\title{
Study of Chemically Induced Variation in Agro-morphological Characters of Wheat Under Heat Stress
}

\author{
S.P. Bharati ${ }^{1}$, Ravi Kant ${ }^{1 *}$, Anil Kumar ${ }^{1}$, V.K. Sharma ${ }^{2}$ and V.K. Choudhary ${ }^{3}$ \\ ${ }^{1}$ Department of Plant Breeding \& Genetics, TCA, Dholi Campus, \\ Muzaffarpur, Bihar-843121, India \\ ${ }^{2}$ Department of Biotechnology \& Molecular Biology, RAU, Pusa, India \\ ${ }^{3}$ Breeder Seed Production Unit, DSF, TCA, Dholi Campus, Muzaffarpur, Bihar-843121, India \\ *Corresponding author
}

\section{A B S T R A C T}

\section{Keywords}

Plant growth regulating

hormones, Agromorphological traits, Normal \&

late sown

conditions, Glycine betaine (600 PPM),

Salicylic acid $(800$ \& 400 PPM),

Ascorbic acid (10

PPM), Tocopherol

(150 PPM),

Elevated

temperature, Heat

stress

Article Info

Accepted:

10 June 2018

Available Online:

10 July 2018
Low productivity (2680 Kg/hectare) (Directorate of Agriculture, Govt. of Bihar; 2012-13) in Bihar is mainly due to late sowing of wheat coupled with prevalent of hot westerly wind during grain filling stage.The wheat production in state can be increased by two means, either by horizontal expansion or by vertical expansion i.e. by using improved variety or use of antioxidant or growth regulators to cope heat stress in delayed sown condition. Mitigation of elevated temperature on late sown wheat through genetic intervention is under way at many research stations. Study of plant growth regulators in overcoming this abiotic stress of elevated temperature during reproductive stage of wheat is of great importance. The present investigation was carried out at DRPCAU, Pusa Bihar during Rabi 2016-2017, with five plant growth regulating hormones viz., Glycine betaine (600 PPM) $\left(\mathrm{T}_{2}\right)$, Salicylic acid (800 PPM) $\left(\mathrm{T}_{3}\right)$, Salicylic acid (400 PPM) $\left(\mathrm{T}_{4}\right)$, Ascorbic acid (10 PPM) $\left(T_{5}\right)$, Tocopherol (150 PPM) $\left(T_{6}\right)$ and control $\left(T_{1}\right)$ on two varieties HD-2733 (normal sown condition) and HD-2985 (late sown condition) with objective to access induced variation and differential influence created on fifteen agro-morphological traits including yield and seed quality attributes. Different statistical tool undertaken for analysis revealed highly significant differences among entries in respect of fifteen different the agro-morphological traits under study after treatments in both conditions i.e. normal and late sown conditions. Treatment $T_{2}$ i.e., Glycine betaine (600 PPM), showed significant effect on highest grain yield per plant via, different agro-morphological character mentioned above for normal and delayed sown condition. Yield enhancement due to effect of Treatment $\mathrm{T}_{2}$ Glycine betaine (600 PPM) was recorded $41.30 \%$ higher under normal sown condition whereas $44.92 \%$ higher under late sown condition in comparison to control. On seed quality traits also showed significant positive effect of Treatment $T_{2}$ Glycine betaine (600 PPM) was observed for seed germination per cent and viability per cent and no effect on vigour index of seed under both normal and delayed sown condition. 


\section{Introduction}

Wheat [Triticum aestivum (L.) em.TheIl] is a thermo sensitive crop mostly grown in temperate environment. In subtropical regions it is cultivated in winter season but it exposed to high temperature stress at the end of the season i.e. at grain filling stage. Heat stress is one of the major limiting factors for growth and productivity in wheat crop particularly in warmer region. Heat stress is anticipated to become more important in future with climate changes, monsoon irregularities and ricewheat cropping system which has compelled wheat crop to be subjected to rapidly ascending temperature coupled with hot dry winds. Most of the crops including wheat exposed to heat stress during later stages of their life cycle (Stone, 2001) i.e. grain filling stage resulting in the development of shriveled grain. Exposure to higher than normal temperature or heat stress reduces yield and decreases quality.

The world acreage under wheat crop is 240 million hectares with production of 758.1 million tones with yield of $2872 \mathrm{~kg} \mathrm{ha}^{-1}$ (FAO, Regional office for Asia and the Pacific, Bangkok, 2015-16). However average yield is quite variable across the world, ranging from less than 1 to over 7 tones $\mathrm{ha}^{-1}$. The differences in per hectare yield are due to differences in the level of inputs, agricultural sophistication and agro-climatic conditions. Bihar accounts for 5.94 MT of wheat production from an area of 2.22 lakh hectare area with a productivity of $2680 \mathrm{Kg} /$ hectare (Directorate of Agriculture, Govt. of Bihar; 2012-13). Low productivity in Bihar is mainly due to late sowing of wheat coupled with prevalent of hot westerly wind during grain filling stage.

Wheat production in Bihar can be increased either by horizontal expansion (cultivation in newly/reclaimed land) or by vertical expansion (using improved variety or use of antioxidant or growth regulators to cope heat stress in delayed sown condition). Scope of horizontal expansion is very limited due to increase in human endeavour other than agriculture by urbanization and industrialization.

Literature on role of plant growth regulating hormones in mitigating heat stress is available. Halim et al., (2006), reported that Salicylic acid (SA) is an endogenous growth regulator of phenolic nature, which participates in the regulation of physiological processes in plants and act as signal molecule in the induction of defence mechanism. Morris et al., 2000 reported that SA modulates secondary metabolites pathway in plant under abiotic stresses whereas Khan et al., (2013) found that it interacts with Proline metabolism and ethylene formation to alleviate the adverse effects of heat stress on photosynthesis in wheat. Wang et al., (2010) reported increase in the activities of different antioxidant enzymes in wheat treated with foliar SA. Similarly, Janda (1999) reported that exogenous SA alleviates the damaging effect of various abiotic stresses and modulates the photosynthetic process of the plant.

According to Smirnoff 2000, Ascorbic acid (AsA) is the most abundant and potential antioxidant known in nature. Ashraf and Foolad 2007 found that exogenous application of AsA enhance the stress tolerance and significantly contribute to the crop production in the stressed environment.

Tocopherol (vitamin E) is synthesized in the plants, mainly concentrated in plastids and acts as an amphiphilic lipid antioxidant. Tocopherol plays a protective role to membrane system in the cell of higher plants (Fryer, 1992; and Wang and Quinn, 2000); assists in maintaining membrane stability (Munné-Bosch and Falk, 2004) and regulates 
the transport of electrons in the photo systemII system (Munné-Bosch and Alegre, 2002).

Therefore, present investigation were under taken to assess the chemically induced variation in agro-morphological characters of wheat under normal and heat stress condition and to analyze the differential influence of chemical application on phenotypic expression of yield attributing characters under heat stress.

\section{Materials and Methods}

The present investigation was carried out in the fields located at Breeder Seed Production Plot in TCA, Dholi, under DRPCAU, Pusa, Bihar during Rabi 2016-2017. The experimental materials of the study comprised of 2 varieties of bread wheat (Triticum aestivum L.) namely HD-2733 and HD-2985 and five plant growth regulator hormones namely Glycine betaine (600 PPM), Salicylic acid (800 PPM), Salicylic acid (400 PPM), Ascorbic acid (10 PPM) and Tocopherol (150 PPM).

The Experiment was sown in Randomized Block Design (RBD) with four replications and two dates of sowing i.e., normal and 30 days delay with two varieties HD-2733 \& HD2985 respectively. In each replication 6 treatment were given namely $\mathrm{T}_{1-\text { control, }} \mathrm{T}_{2}$ Glycinebetaine (600 PPM), $\mathrm{T}_{3}$-Salicylic acid (800 PPM), $\mathrm{T}_{4}$-Salicylic acid (400 PPM), $\mathrm{T}_{5^{-}}$ Ascorbic acid (10 PPM), $\mathrm{T}_{6}$-Tocopherol (150 PPM)at two stages i.e., first at vegetative and second at reproductive stage of plant. Standard agronomic practices were adopted with $22 \mathrm{~cm}$ row to row spacing. Five random plants per replication were tagged to record observations on twelve seed yield i.e. Days to flowering, Days to maturity, Plant height $(\mathrm{cm})$, Pollen fertility per cent, Spikelet fertility per cent, Flag leaf area $\left(\mathrm{cm}^{2}\right)$, Number of tillers per plant, Ear length $(\mathrm{cm})$,
Number of grains per ear, 1000 grain weight (g), Harvest index, Seed yield per plant (g) and yield attributing characters along with three seed quality traits i.e. Seed germination per cent, Vigour Index, Viability per cent.

The statistical analysis of the data on individual character was carried out on the mean values over four replications. The statistical methods adopted were Mean. Range, Coefficient of Variation, Analysis of variance (ANOVA) for all characters, Correlation coefficient, Path coefficient analysis and Simple Regression coefficient analysis.

\section{Results and Discussion}

Analysis of variance revealed highly significant differences among entries in respect of all the agro-morphological traits under studied in both conditions i.e. normal and delayed sown condition except for number of tiller per plant in delayed sown condition.

Effect of different plant growth regulating hormones on mean performance of fifteen agro-morphological traits under normal sown condition showed varied response such as:

For days to flowering, $\mathrm{T}_{1}$ showed the longest duration of 72.05 days under normal sown condition, whereas $\mathrm{T}_{6}$ acquired the shortest duration of 66.10 days. For days to maturity, $\mathrm{T}_{2}$ recorded shortest duration of 109.32 days followed by $\mathrm{T}_{3}$ (111.62 days) and $\mathrm{T}_{5}$ (111.02 days). For plant height, $\mathrm{T}_{6}$ recorded maximum $(99.31 \mathrm{~cm})$, whereas $T_{2}$ recorded shortest plant height . The maximum pollen fertility per cent was exhibited by $\mathrm{T}_{2}$ (77.93) and minimum pollen fertility per cent was exhibited by $T_{1}$ (69.71). For spikelet fertility per cent, $\mathrm{T}_{6}$ recorded highest $(90.95 \%)$, whereas $\mathrm{T}_{2}$ recorded lowest value. For flag leaf area, $\mathrm{T}_{1}$ and $\mathrm{T}_{3}$ showed highest and lowest value respectively. For number of tillers per plant $T_{2}$ 
showed maximum tillers whereas $T_{1}$ recorded minimum number of tillers. $T_{2}$ recorded highest ear length. For number of grains per ear, the maximum value being associated with $\mathrm{T}_{2}$ while minimum value recorded with the $\mathrm{T}_{1}$. The treatment $\mathrm{T}_{2}$ recorded highest 1000 grain weight (46.61) followed by $\mathrm{T}_{6}$ (43.34).Similarly for harvest index, the highest significant effect being associated with $\mathrm{T}_{2}$ (48.63\%) in compare with control. For seed yield per plant the lowest and highest effect being associated with $T_{1}(14.36 \mathrm{~g})$ and $T_{2}$ $(20.29 \mathrm{~g})$, respectively.

Correlation coefficient analysis of fifteen agro-morphological traits under normal sowing condition reveals that seed yield per plant exhibited highest positive and significant association with seed germination percentage (0.5783) followed by harvest index (0.5216), 1000 grain weight and number of tillers per plant.

The cause and effect analysis reveals that 1000 grain weight showed maximum direct positive phenotypic effect $(0.6378)$ on yield per plant whereas days to flowering had minimum (0.1816) direct effect. The direct effects of other yield components, viz., number of tillers per plant, pollen fertility per cent, spikelet fertility per cent and number of grain per year on yield per plant were 0.4457 , $0.3705,0.2809$ and 0.2146 respectively. Ear length, harvest index, days to maturity, plant height, and flag leaf area showed negative direct effects $(-0.9637,-0.4253$, $0.3302,-0.1296$, and -0.0793 , respectively) on seed yield per plant.

The adjusted coefficient of determination $\left(\mathrm{R}^{2}\right)$ for variance of seed yield was recorded as 93.39 per cent. The traits like, days to maturity, pollen fertility per cent, spikelet fertility per cent, number of tiller per plant, ear length, 1000 grain weight, harvest index, seed germination per cent and seed viability per cent (0.9393) recorded maximum values of
$\mathrm{R}^{2}$. Among fifteen agro-morphological traits taken for study of effect of plant growth regulator chemicals under normal sown condition, five traits viz., spikelet fertility per cent, number of tiller per plant, ear length, 1000 grain weight and harvest index was found significant indicated the effect of these traits on seed yield.

Effect of different plant growth regulating hormones on mean performance of fifteen agro-morphological traits under delayed sown condition showed varied response such as:

For days to flowering $\mathrm{T}_{6}$ results in significant earlier days to flowering than check $\left(T_{1}\right)$. For the traits days to maturity, $\mathrm{T}_{6}$ recorded shortest days (99.87 days) followed by $\mathrm{T}_{5}$ (100.27days).For plant height, $T_{2}$ recorded maximum $(93.13 \mathrm{~cm})$, whereas treatment $\left(\mathrm{T}_{1}\right)$ recorded shortest plant height. The maximum pollen fertility per cent was exhibited by $\mathrm{T}_{6}$ (75.27) and minimum pollen fertility per cent was exhibited by $\mathrm{T}_{1}(67.72 \%)$. Effect of treatment on spikelet fertility per cent, $\mathrm{T}_{2}$ recorded highest $(87.03 \%)$, whereas $\mathrm{T}_{4}$ recorded lowest spikelet fertility per cent $(84.11 \%)$. For flag leaf area $\mathrm{T}_{3}$ and $\mathrm{T}_{1}$ showed highest and lowest value respectively. Number of tillers per plant also influenced maximum by treatment $\mathrm{T}_{2}$ whereas $\mathrm{T}_{1}$ recorded minimum value for traits. For ear length effect of treatment $\mathrm{T}_{2}$ showed recorded maximum value, whereas $T_{1}$ recorded minimum value. For number of grains per ear the maximum value being associated with $T_{2}$, while minimum value recorded with $\mathrm{T}_{1}$. The treatment $\mathrm{T}_{2}$ recorded highest 1000 grain weight $(44.24 \mathrm{~g})$ followed by $\mathrm{T}_{1}(34.68 \mathrm{~g})$. For harvest index the highest significant effect being associated with the treatment $\mathrm{T}_{2}$ (47.47\%) in compare with control. Effect of treatment $\mathrm{T}_{2}$ Glycine betaine (600 PPM) $(17.13 \mathrm{~g})$ also influenced seed yield per plant significantly in compare with control $\mathrm{T}_{1}$ (11.82g) (Table 1-9). 
Table.1 Analysis of variance for fifteen agro-morphological characters under normal and delayed sowing conditions

\begin{tabular}{|c|c|c|c|c|c|c|c|}
\hline & \multirow[t]{2}{*}{ Characters } & \multicolumn{3}{|c|}{ Mean sum of squares (normal) } & \multicolumn{3}{|c|}{ Mean sum of squares (delayed) } \\
\hline & & $\begin{array}{c}\text { Rep. } \\
(\mathbf{d} \mathbf{f}=\mathbf{3})\end{array}$ & $\begin{array}{l}\text { Treatment } \\
(\mathrm{d} \mathbf{f}=\mathbf{5})\end{array}$ & $\begin{array}{c}\text { Error } \\
(\mathrm{d} \mathbf{f}=\mathbf{1 5})\end{array}$ & $\begin{array}{c}\text { Rep. } \\
(\mathbf{d} \mathbf{f = 3})\end{array}$ & $\begin{array}{l}\text { Treatment } \\
(\mathrm{d} \mathbf{f}=5)\end{array}$ & $\begin{array}{c}\text { Error } \\
(\mathrm{d} \mathbf{f}=15)\end{array}$ \\
\hline 1. & Days to Flowering & 4.43 & $18.42 *$ & 5.66 & 10.16 & $54.1 * *$ & 11.56 \\
\hline 2. & Day to Maturity & 1.83 & $11.70 * *$ & 3.20 & 2.84 & $10.23 *$ & 4.24 \\
\hline 3. & Plant height & 4.34 & $25.14 *$ & 7.72 & 7.72 & $42.76^{*}$ & 12.28 \\
\hline 4. & Pollen fertility per cent & 21.17 & $33.78 *$ & 9.66 & 15.85 & $33.5 * *$ & 8.71 \\
\hline 5. & Spikelet fertility per cent & 3.13 & 4.77 & 3.70 & 15.92 & $4.34^{*}$ & 6.11 \\
\hline 6. & Flag leaf Area $(\mathrm{cm} 2)$ & 0.052 & $1.08^{*}$ & 0.42 & 2.20 & $1.46^{*}$ & 0.49 \\
\hline 7. & Number of tiller Per Plant & 0.19 & $1.32 * *$ & 0.23 & 1.52 & 1.43 & 0.67 \\
\hline 8. & Ear length $(\mathrm{cm})$ & 1.38 & $9.90 * *$ & 1.60 & 2.24 & $10.5^{* *}$ & 1.69 \\
\hline 9. & Number of grain per ear & 7.48 & $67.63 * *$ & 15.73 & 1.17 & $23.70^{*}$ & 7.83 \\
\hline 10. & 1000 grain weight (gm) & 13.45 & $70.77 * *$ & 9.47 & 8.22 & $51.47 * *$ & 11.08 \\
\hline 11. & Harvest Index per cent & 8.71 & $56.17 * *$ & 13.59 & 14.85 & $55.60 *$ & 15.66 \\
\hline 12. & Seed yield per plant & 10.24 & $20.75 *$ & 6.60 & 5.86 & $16.3 * *$ & 3.85 \\
\hline 13. & Seed germination per cent & 3.72 & $19.15 *$ & 5.69 & 3.56 & $18.8 * *$ & 4.67 \\
\hline 14. & Seed viability per cent & 4.17 & $15.73 * *$ & 4.35 & 2.98 & $22.9 * *$ & 5.97 \\
\hline 15. & Vigour Index & 5971.12 & $124173.12 * *$ & 16954.24 & 10797.75 & 23893.26 & 19780.92 \\
\hline
\end{tabular}


Table.2 Mean performance of fifteen agro-morphological traits under normal sowing conditions

\begin{tabular}{|c|c|c|c|c|c|c|c|c|c|c|c|c|c|c|c|c|}
\hline SN & reat. & DF & DM & PH & PFP & SFP & FLA & TPP & EL & GPE & TGW & HI & SYPP & SGP & VP & VI \\
\hline 1 & $\mathrm{~T} 1$ & 72.05 & 114.52 & 98.37 & 69.71 & 88.72 & 19.96 & 5.81 & 8.86 & 39.39 & 35.91 & 38.37 & 14.36 & 89.80 & 86.64 & 1366.04 \\
\hline 2 & $\mathrm{~T} 2$ & 69.24 & $109.32 *$ & $92.98 *$ & 77.93* & 87.92 & 19.02 & $7.42 *$ & $13.77 *$ & $50.49 *$ & $46.61 *$ & $48.63^{*}$ & $20.29 *$ & 96.04* & $92.03 *$ & 1478.95 \\
\hline 3 & 73 & 69.67 & $111.62 *$ & 95.25 & $76.06^{*}$ & $0.12 *$ & 18.72 & $6.56^{*}$ & $10.97 *$ & 41.65 & 38.86 & 40.86 & 14.92 & 92.47 & $90.84^{*}$ & 1385.16 \\
\hline 4 & $\Gamma 4$ & 71.78 & 112.25 & 99.05 & 74.02 & $9.04 *$ & 9.87 & $7.09 *$ & $11.20 *$ & 42.80 & 36.05 & 38.75 & 4.54 & 91.24 & $90.97 *$ & 1376.16 \\
\hline 5 & T5 & 69.71 & $111.02 *$ & 97.99 & $75.10^{*}$ & $88.92 *$ & 19.85 & 6.27 & 10.71 & 40.61 & 40.35 & 40.57 & 15.66 & 92.98 & $91.26^{*}$ & $1705.33^{*}$ \\
\hline \multirow[t]{4}{*}{6} & T6 & $66.10 *$ & 112.30 & 99.31 & 76.90* & $90.95^{*}$ & 19.24 & $6.53 *$ & $11.35^{*}$ & $46.07 *$ & $43.34 *$ & 42.44 & 17.28 & $94.17 *$ & $91.75^{*}$ & $1758.80^{*}$ \\
\hline & Mean & 69.76 & 111.84 & 97.16 & 74.95 & 89.26 & 19.44 & 6.61 & 11.14 & 43.50 & 40.19 & 41.60 & 16.17 & 92.78 & 90.58 & 1511.75 \\
\hline & $\mathrm{Cv}$ & 3.41 & 1.60 & 2.86 & 4.14 & 2.15 & 3.36 & 7.35 & 11.37 & 9.11 & 7.65 & 8.86 & 15.88 & 2.57 & 2.30 & 8.61 \\
\hline & $\mathrm{CD}$ & 3.58 & 2.69 & 4.18 & 4.6 & 3.45 & 2.34 & 0.73 & 1.91 & 5.97 & 4.63 & 5.55 & 3.87 & 3.59 & 3.14 & 196.24 \\
\hline
\end{tabular}

Table.3 Mean performance for fifteen agro-morphological traits under delayed sowing conditions

\begin{tabular}{|c|c|c|c|c|c|c|c|c|c|c|c|c|c|c|c|c|}
\hline SN & Treat. & DF & DM & PH & PFP & SFP & FLA & TPP & EL & GPE & TGW & HI & SYPP & SGP & VP & VI \\
\hline 1 & $\mathrm{~T}_{1}$ & 68.37 & 102.72 & 83.91 & 67.72 & 84.98 & 17.15 & 5.35 & 7.73 & 38.51 & 34.68 & 36.87 & 11.82 & 88.61 & 84.78 & 1224.72 \\
\hline 2 & $\mathrm{~T}_{2}$ & 64.44 & $101.19 *$ & 93.13 & 71.92 & $87.03 *$ & 18.11 & $6.80 *$ & $12.75^{*}$ & $44.55^{*}$ & $44.24 *$ & $47.47^{*}$ & $17.13^{*}$ & $94.99 *$ & $91.45^{*}$ & 1166.55 \\
\hline 3 & $\mathrm{~T}_{3}$ & 64.98 & $102.31 *$ & 88.56 & $74.65^{*}$ & 85.41 & $18.81 *$ & $5.75^{*}$ & $10.02 *$ & 40.38 & 37.58 & 39.74 & 12.28 & 91.71 & 88.31 & 1202.44 \\
\hline 4 & $\mathrm{~T}_{4}$ & 67.55 & 104.09 & 87.08 & $73.87 *$ & 84.11 & 17.60 & $5.54 *$ & $9.97 *$ & 39.91 & 36.08 & 37.86 & 12.10 & 90.97 & 87.15 & $1347.64 *$ \\
\hline 5 & $\mathrm{~T}_{5}$ & $60.29 *$ & $100.27 *$ & 89.38 & $75.07 *$ & $85.66 *$ & 18.06 & $6.05 *$ & $10.22 *$ & 40.94 & 38.53 & 39.93 & 13.40 & $92.87 *$ & $89.29 *$ & $1350.39 *$ \\
\hline \multirow[t]{3}{*}{6} & $\mathrm{~T}_{6}$ & $59.39 *$ & 99.87* & 91.56 & $75.27 *$ & $86.46^{*}$ & 17.34 & $6.68 *$ & $10.89 *$ & $44.21 *$ & $41.77 *$ & 40.41 & 14.51 & $93.09 *$ & $90.42 *$ & $1228.60 *$ \\
\hline & Mean & 64.17 & 101.74 & 88.94 & 73.08 & 85.61 & 17.84 & 6.03 & 10.26 & 41.42 & 38.81 & 40.38 & 13.54 & 92.04 & 88.57 & 1253.39 \\
\hline & $\mathrm{Cv}$ & 5.29 & 2.02 & 3.94 & 4.04 & 2.88 & 3.94 & 13.59 & 12.67 & 6.75 & 8.57 & 9.80 & 14.49 & 2.34 & 2.76 & 11.22 \\
\hline
\end{tabular}


Table.4 Correlated effect of chemical application (Normal)

\begin{tabular}{|c|c|c|c|c|c|c|c|c|c|c|c|c|c|c|}
\hline & DF & DM & PH & PFP & SFP & FLA & TPP & EL & GPE & TGW & HI & SGP & VP & VI \\
\hline \multicolumn{15}{|l|}{ DF } \\
\hline DM & 0.2016 & & & & & & & & & & & & & \\
\hline PH & -0.0320 & $0.4059 *$ & & & & & & & & & & & & \\
\hline PF & $\begin{array}{c}- \\
0.5056^{*}\end{array}$ & -0.3434 & -0.0876 & & & & & & & & & & & \\
\hline SFP & -0.2545 & 0.1571 & 0.0808 & 0.2859 & & & & & & & & & & \\
\hline FLA & $0.4191^{*}$ & $0.5492 * *$ & 0.3774 & -0.4037 & 0.0551 & & & & & & & & & \\
\hline TPP & -0.0751 & -0.2980 & - & 0.3446 & -0.051 & -0.2806 & & & & & & & & \\
\hline EL & -0.3244 & $\begin{array}{c}- \\
0.6302 * *\end{array}$ & -0.2843 & 0.4019 & 0.2440 & $0.4680 *$ & $0.5038^{*}$ & & & & & & & \\
\hline GPE & $\begin{array}{c}- \\
0.4781 *\end{array}$ & -0.3895 & -0.2400 & $0.4740 *$ & $\begin{array}{c}- \\
0.0465\end{array}$ & -0.3569 & $0.4213^{*}$ & $0.5533 * *$ & & & & & & \\
\hline TGW & $\begin{array}{c}- \\
0.4615^{*}\end{array}$ & $-0.4369 *$ & -0.2333 & $0.4345 *$ & 0.1567 & $0.4642 *$ & 0.3086 & $0.7416 * *$ & $0.4394 *$ & & & & & \\
\hline HI & -0.0533 & -0.3810 & $\begin{array}{c}- \\
0.4623 *\end{array}$ & 0.2326 & 0.0744 & -0.2202 & $0.4659 *$ & 0.3089 & $0.5122 *$ & $0.5229 * *$ & & & & \\
\hline SGP & -0.1775 & -0.3284 & -0.3023 & 0.1584 & 0.0502 & 0.1493 & $0.4492 *$ & $0.4835^{*}$ & 0.2851 & $0.6508 * *$ & $0.6710 * *$ & & & \\
\hline VP & -0.3711 & -0.3431 & -0.1350 & $0.4774 *$ & $\begin{array}{c}- \\
0.2794\end{array}$ & -0.1316 & $0.4793^{*}$ & $0.4443^{*}$ & 0.2156 & 0.3545 & 0.2341 & $0.4332 *$ & & \\
\hline VI & -0.3636 & -0.0638 & 0.2858 & 0.1304 & $\begin{array}{c}- \\
0.1320\end{array}$ & 0.0604 & 0.0440 & 0.1087 & 0.1881 & 0.3647 & 0.2092 & $0.4246^{*}$ & 0.3270 & \\
\hline SYPP & -0.0964 & -0.3114 & -0.3627 & 0.3319 & 0.1663 & 0.1526 & $0.4468 *$ & 0.2653 & 0.2589 & $0.4911^{*}$ & $0.5216 * *$ & $0.5783 * *$ & 0.1561 & 0.245 \\
\hline
\end{tabular}


Table.5 Correlated effect of chemical application (Late)

\begin{tabular}{|c|c|c|c|c|c|c|c|c|c|c|c|c|c|c|}
\hline & DF & DM & PH & PFP & SFP & FLA & TPP & EL & GPE & TGW & HI & SGP & VP & VI \\
\hline \multicolumn{15}{|l|}{ DF } \\
\hline DM & $0.5798 * *$ & & & & & & & & & & & & & \\
\hline PH & $-0.4224^{*}$ & -0.2720 & & & & & & & & & & & & \\
\hline PF & -0.2488 & -0.2884 & 0.2665 & & & & & & & & & & & \\
\hline SFP & -0.1997 & 0.1400 & 0.1982 & 0.1082 & & & & & & & & & & \\
\hline FLA & 0.1249 & 0.0788 & 0.1720 & -0.2272 & 0.0804 & & & & & & & & & \\
\hline TPP & -0.2712 & -0.1332 & 0.3693 & $\begin{array}{c}- \\
0.7438 * *\end{array}$ & 0.0987 & $\begin{array}{c}- \\
0.5836 * *\end{array}$ & & & & & & & & \\
\hline EL & -0.3089 & -0.0393 & $0.6104 * *$ & -0.1578 & 0.2145 & $0.4554^{*}$ & $0.6239 * *$ & & & & & & & \\
\hline GPE & -0.3712 & -0.1525 & $0.7253 * *$ & 0.1396 & $0.5419 * *$ & 0.0910 & $0.4922^{*}$ & $0.6141 * *$ & & & & & & \\
\hline TGW & -0.2663 & -0.0335 & $0.5664 * *$ & -0.1229 & 0.2895 & -0.2875 & $0.5258 * *$ & $0.7078 * *$ & $0.4143^{*}$ & & & & & \\
\hline HI & -0.1877 & -0.1694 & 0.3990 & 0.0738 & 0.0576 & -0.0168 & 0.3314 & 0.3517 & 0.1834 & $0.5196 * *$ & & & & \\
\hline SGP & -0.3651 & -0.3579 & $0.5606 * *$ & 0.3940 & 0.2188 & 0.1919 & 0.2627 & $0.6540 * *$ & $0.5688 * *$ & $0.4688 *$ & 0.3023 & & & \\
\hline VP & $-0.4799 *$ & $-0.4602 *$ & $0.7145^{* *}$ & $0.5093^{*}$ & 0.3131 & -0.2669 & 0.2981 & $0.5490 * *$ & $0.5410 * *$ & $0.5333 * *$ & 0.3134 & $0.6868 * *$ & & \\
\hline VI & 0.0810 & -0.0835 & -0.0391 & -0.1459 & -0.2946 & 0.1346 & -0.1721 & -0.0677 & -0.2113 & -0.1255 & $-0.4283^{*}$ & 0.0201 & 0.0441 & \\
\hline SYPP & -0.0868 & -0.2191 & $0.4141 *$ & 0.0017 & 0.0286 & 0.0348 & 0.3581 & 0.3740 & 0.3934 & $0.5149 * *$ & $0.6622 * *$ & 0.3328 & 0.2234 & 0.1668 \\
\hline
\end{tabular}


Table.6 Result of cause and effect - analysis of chemical application under normal condition

\begin{tabular}{|c|c|c|c|c|c|c|c|c|c|c|c|c|c|c|}
\hline & DF & DM & PH & PFP & SFP & FLA & TPP & EL & GPE & TGW & HI & SGP & VP & VI \\
\hline DF & 0.1816 & 0.0366 & -0.0058 & -0.0918 & 0.0462 & -0.0761 & -0.0136 & -0.0589 & -0.0868 & -0.0838 & -0.0097 & -0.0322 & -0.0674 & -0.0661 \\
\hline DM & -0.0666 & -0.3302 & -0.1340 & 0.1134 & 0.0518 & 0.1813 & 0.0984 & 0.2081 & 0.1286 & 0.1442 & 0.1258 & 0.1084 & 0.1133 & 0.0211 \\
\hline PH & 0.0041 & -0.0525 & -0.1294 & 0.0113 & 0.0104 & 0.0488 & 0.0624 & 0.0368 & 0.0311 & 0.0302 & 0.0598 & 0.0391 & 0.0175 & -0.0370 \\
\hline PF & -0.1873 & -0.1272 & -0.0324 & 0.3705 & -0.1059 & 0.1496 & 0.1277 & 0.1489 & 0.1756 & 0.1610 & 0.0862 & 0.0587 & 0.1769 & 0.0483 \\
\hline SFP & 0.0715 & -0.0441 & -0.0227 & -0.0803 & 0.2809 & -0.0155 & -0.0146 & 0.0685 & -0.0131 & 0.0440 & 0.0209 & 0.0141 & -0.0785 & -0.0371 \\
\hline FLA & 0.0332 & 0.0435 & 0.0299 & -0.0320 & 0.0043 & -0.0793 & -0.0114 & -0.0371 & -0.0283 & -0.0368 & -0.0175 & -0.0118 & -0.0104 & 0.0048 \\
\hline TPP & -0.0335 & -0.1328 & -0.2149 & 0.1536 & -0.0231 & 0.0643 & 0.4457 & 0.2245 & 0.1878 & 0.1375 & 0.2077 & 0.2002 & 0.2136 & 0.0196 \\
\hline EL & 0.3126 & 0.6074 & 0.2740 & -0.3873 & -0.2351 & -0.4510 & -0.4855 & -0.9637 & -0.5332 & -0.7146 & -0.2977 & -0.4660 & -0.4282 & -0.1047 \\
\hline GPE & -0.1026 & -0.0836 & -0.0515 & 0.1017 & -0.0099 & 0.0766 & 0.0904 & 0.1187 & 0.2146 & 0.0943 & 0.1099 & 0.0612 & 0.0463 & 0.0404 \\
\hline TGW & -0.2943 & -0.2786 & -0.1488 & 0.2771 & 0.0999 & 0.2961 & 0.1968 & 0.4730 & 0.2802 & 0.6378 & 0.3335 & 0.4151 & 0.2261 & 0.2327 \\
\hline HI & 0.0226 & 0.1620 & 0.1966 & -0.0989 & -0.0316 & -0.0937 & -0.1982 & -0.1314 & -0.2178 & -0.2224 & -0.4253 & -0.2854 & -0.0996 & -0.0890 \\
\hline SGP & -0.0969 & -0.1792 & -0.1650 & 0.0864 & 0.0273 & 0.0814 & 0.2451 & 0.2638 & 0.1556 & 0.3551 & 0.3661 & 0.5456 & 0.2363 & 0.2316 \\
\hline VP & 0.0762 & 0.0704 & 0.0277 & -0.0980 & 0.0573 & -0.0270 & -0.0984 & -0.0912 & -0.0443 & -0.0728 & -0.0480 & -0.0889 & -0.2053 & -0.0671 \\
\hline VI & -0.0173 & -0.0030 & 0.0136 & 0.0062 & -0.0062 & -0.0029 & 0.0021 & 0.0052 & 0.0089 & 0.0173 & 0.0099 & 0.0202 & 0.0155 & 0.0475 \\
\hline SYPP & -0.0964 & -0.3114 & -0.3627 & 0.3319 & 0.1663 & 0.1526 & 0.4468 & 0.2653 & 0.2589 & 0.4911 & 0.5216 & 0.5783 & 0.1561 & 0.2450 \\
\hline $\mathbf{R}^{2}$ & \multicolumn{14}{|c|}{0.6755} \\
\hline
\end{tabular}


Table.7 Result of cause and effect - analysis of chemical application under delayed condition

\begin{tabular}{|c|c|c|c|c|c|c|c|c|c|c|c|c|c|c|}
\hline & DF & DM & PH & PFP & SFP & FLA & TPP & EL & GPE & TGW & HI & SGP & VP & VI \\
\hline DF & 0.2236 & 0.1296 & -0.0944 & 0.0556 & -0.0446 & -0.0279 & -0.0606 & -0.0691 & -0.0830 & -0.0595 & -0.0420 & -0.0816 & -0.1073 & -0.0181 \\
\hline DM & -0.1793 & -0.3093 & 0.0841 & -0.0892 & -0.0433 & 0.0244 & 0.0412 & 0.0122 & 0.0472 & 0.0104 & 0.0524 & 0.1107 & 0.1423 & -0.0258 \\
\hline PH & 0.1330 & 0.0856 & -0.3149 & 0.0839 & -0.0624 & 0.0542 & -0.1163 & -0.1922 & -0.2284 & -0.1784 & -0.1257 & -0.1765 & -0.2250 & -0.0123 \\
\hline $\mathbf{P F}$ & 0.0027 & 0.0032 & -0.0029 & 0.011 & -0.0012 & 0.0025 & 0.0013 & -0.0017 & -0.0015 & -0.0014 & -0.0008 & -0.0044 & -0.0056 & 0.0016 \\
\hline SFP & 0.0525 & -0.0368 & -0.0521 & 0.0284 & -0.2627 & 0.0211 & -0.0259 & -0.0564 & -0.1424 & -0.0761 & -0.0151 & -0.0575 & -0.0822 & -0.0774 \\
\hline FLA & -0.0023 & -0.0014 & -0.0031 & 0.0041 & -0.0015 & 0.0183 & -0.0047 & -0.0083 & -0.0017 & -0.0053 & 0.0003 & -0.0035 & -0.0049 & 0.0025 \\
\hline TPP & 0.0521 & 0.0256 & -0.0710 & -0.0223 & -0.0190 & 0.0495 & -0.1922 & -0.1199 & -0.0946 & -0.1011 & -0.0637 & -0.0505 & -0.0573 & -0.0331 \\
\hline EL & 0.0256 & 0.0033 & -0.0505 & 0.0131 & -0.0177 & 0.0377 & -0.0516 & -0.0827 & -0.0508 & -0.0586 & -0.0291 & -0.0541 & -0.0454 & -0.0056 \\
\hline GPE & -0.3235 & -0.1329 & 0.6320 & -0.1216 & 0.4722 & -0.0793 & 0.4289 & 0.5351 & 0.8715 & 0.3611 & 0.1599 & 0.4957 & 0.4714 & 0.1841 \\
\hline TGW & -0.1310 & -0.0165 & 0.2788 & -0.0605 & 0.1425 & -0.1415 & 0.2588 & 0.3483 & 0.2039 & 0.4922 & 0.2557 & 0.2307 & 0.2625 & 0.0618 \\
\hline HI & -0.1276 & -0.1152 & 0.2712 & -0.0502 & 0.0392 & 0.0114 & 0.2253 & 0.2390 & 0.1247 & 0.3532 & 0.6797 & 0.2055 & 0.2130 & 0.2911 \\
\hline SGP & 0.0328 & 0.0321 & -0.0503 & 0.0354 & -0.0196 & 0.0172 & -0.0236 & -0.0587 & -0.0511 & -0.0421 & -0.0271 & -0.0898 & -0.0616 & 0.0018 \\
\hline VP & 0.1372 & 0.1316 & -0.2043 & 0.1456 & -0.0895 & 0.0763 & -0.0852 & -0.1570 & -0.1547 & -0.1525 & -0.0896 & -0.1964 & -0.2859 & 0.0126 \\
\hline VI & 0.0175 & -0.0181 & -0.0085 & -0.0316 & -0.0638 & -0.0291 & -0.0372 & -0.0146 & -0.0457 & -0.0272 & -0.0927 & 0.0043 & 0.0095 & 0.2164 \\
\hline SYPP & -0.0868 & -0.2191 & 0.4141 & 0.0017 & 0.0286 & 0.0348 & 0.3581 & 0.3740 & 0.3934 & 0.5149 & 0.6622 & 0.3328 & 0.2234 & 0.1668 \\
\hline $\mathbf{R}^{2}$ & \multicolumn{14}{|c|}{0.7279} \\
\hline
\end{tabular}


Table.8 Simple Regression coefficient on seed yield of different chemically induced traits under timely sown condition

\begin{tabular}{|c|c|c|c|c|c|}
\hline Character & Beta & $\begin{array}{c}\mathbf{R} \\
\text { square }\end{array}$ & $\begin{array}{c}\text { Reg. } \\
\text { Coeff. }\end{array}$ & $\begin{array}{c}\text { Std } \\
\text {.Error }\end{array}$ & Significance \\
\hline D M & $\begin{array}{c}- \\
0.3003\end{array}$ & 0.0935 & $\begin{array}{c}- \\
0.4336\end{array}$ & 0.3282 & \\
\hline P F P & 0.3490 & 0.1158 & 0.2746 & 0.1748 & \\
\hline S F P & $\begin{array}{c}- \\
0.2862\end{array}$ & 0.0477 & $\begin{array}{c}- \\
0.4640\end{array}$ & 0.3165 & $*$ \\
\hline T PP & 0.5045 & 0.2254 & 2.4393 & 1.1047 & $* *$ \\
\hline E L & $\begin{array}{c}- \\
0.8103\end{array}$ & $\begin{array}{c}- \\
0.2149\end{array}$ & $\begin{array}{c}- \\
1.4041\end{array}$ & 0.6220 & $*$ \\
\hline T G W & 0.4722 & 0.2319 & 0.3116 & 0.2217 & $* *$ \\
\hline H I & $\begin{array}{c}- \\
0.2207\end{array}$ & $\begin{array}{c}- \\
0.1151\end{array}$ & $\begin{array}{c}- \\
0.1493\end{array}$ & 0.1773 & $*$ \\
\hline S G P & 0.5286 & 0.3057 & 0.5824 & 0.3061 & \\
\hline S V P & $\begin{array}{c}- \\
0.2600\end{array}$ & $\begin{array}{c}- \\
0.0406\end{array}$ & $\begin{array}{c}- \\
0.3177\end{array}$ & 0.2723 & \\
\hline \multicolumn{3}{|c|}{$\mathbf{R}^{2}$} & \multicolumn{3}{|c|}{0.9393} \\
\hline
\end{tabular}

Table.9 Simple Regression coefficient on seed yield of different chemically induced traits under delayed sown condition

\begin{tabular}{|c|c|c|c|c|c|}
\hline Character & Beta & R square & Reg. Coeff. & Std .Error & significance \\
\hline DF F & 0.283 & -0.0225 & 0.163 & 0.107 & \\
\hline D M & -0.385 & 0.084 & -0.434 & 0.219 & \\
\hline G P E & 0.483 & 0.190 & 0.391 & 0.156 & $*$ \\
\hline T G W & 0.321 & 0.165 & 0.190 & 0.118 & $*$ \\
\hline H I & 0.627 & 0.415 & 0.333 & 0.101 & $*$ \\
\hline S V P & -0.456 & -0.102 & -0.391 & 0.187 & $*$ \\
\hline S V I & 0.209 & -0.035 & 0.004 & 0.003 & \\
\hline & & 0.9035 & & & \\
\hline
\end{tabular}

Correlation studies revealed that grain yield per plant showed significant positive correlation with plant height, 1000 grain weight and harvest index under delayed sown condition.

Study of cause and effect analysis under delayed sown condition also revealed that grain yield per plant were directly positive associated with days to flowering, pollen fertility per cent, flag leaf area, number of grain per ear, 1000 grain weight, harvest index and vigour index.

The regression of seed yield per plant on fifteen agro-morphological traits like, days to 
flowering, days to maturity, number of grain per ear,1000 grain weight, harvest index and viability per cent recorded maximum values of $\mathrm{R}^{2}(0.9035)$. Hence, selection of plant growth regulating hormones based on these characters would be more effective for yield improvement.

In conclusion, out of five plant growth regulating hormones, the effect of treatment $\mathrm{T}_{2}$ Glycine betaine (600 PPM), showed significant effect on highest grain yield per plant via, different agro-morphological character mentioned above for both normal and delayed condition.

Yield enhancement due to effect of treatment $\mathrm{T}_{2}$ Glycine betaine (600 PPM) was recorded $41.30 \%$ higher under normal sown condition whereas $44.92 \%$ higher under delayed sown condition in comparison to control T1.

Seed quality traits also showed significant positive effect of Glycine betaine (600 PPM) for seed germination per cent and viability per cent and no effect on vigour index of seed under both normal and delayed sown condition.

\section{Research highlight}

Significant yield enhancement due to effect of Glycine betaine (600 PPM) (Plant growth regulating hormone) accomplish with positive effect on seed quality traits such as germination, viability \& vigour were recorded under both normal sown condition as well as under late sown condition in comparison to control.

\section{Acknowledgement}

Financial help received during conduct of experiment as fellowship and for purchase of chemical molecules from DRPCAU, Pusa is duly acknowledged.

\section{References}

Fryer MJ (1992). The antioxidant effects of thylakoid vitamin E ( $\alpha$-tocopherol). Plant Cell Environ.,15: 381-392.

Janda, T., G. Szalai, I. Tari and E-Paldi. 1999. Hydroponic treatment with salicylic acid decreases the effect of chilling injury in maize (Zea mays L.) Plants.Planta,208: 175-180.

Khan, M.I., N. Iqbal, A. Masood, T.S. Per and N.A. Khan(2013). Salicylic acid alleviates adverse effects of heat stress on photosynthesis through changes in proline production and ethylene formation. Plant Signal Behav. 8(11): 263-74.

M. Ashraf, and M. R. Foolad, 'Roles of Glycine Betaine and Proline in Improving Plant Abiotic Stress Resistance', Environmental \& Experimental Botany, 59 (2007): 20616

Munne - Bosch (2005).The role of alpha Tocopherol in plant stress tolerance. $J$ Plant Physiol. 162(7):743.8.

Munne- Bosch and Jon Falk (2004).Tocopherols plants, biosynthesis, tocotrienoils, antioxidents, chloroplast, vitamin E, lipids, biochemical pathways, enzyme. Insights into the function of Tocopherols in plants, 218:323- 326.

Munné-Bosch S, Alegre L (2002). The function tocopherols and to cotrienols in plants. Crit. Rev. Plant Sci., 21: 3157.

Smirnoff, N. and G.L. Wheeler, 2000. Ascorbic acid in plants: Biosynthesis and function. Crit. Rev. Plant Sci., 19: 267-290 .

Stone, P. (2001). The effects of heat stress on cereal yield and quality. In: A.S. Basra (Ed.) Crop

V.A. Halim., A. Vess., D. Scheel., S. Rosahl. (2006). The role of salicylic acid and 
jasmonic acid in pathogen defence. 8: 307-313.

V.A. Halim., A. Vess., D. Scheel., S. Rosahl. (2006) The role of salicylic acid and jasmonic acid in pathogen defence .Vol:08, page - 307- 313.

Wang, L.J., Fan, L., Loescher, W., Duan, W., Liu, G.J. and Cheng, J.S. 2010.
Salicylic acid alleviates decreases in photosynthesis under heat stress and accelerates recovery in grapevine leaves. BMC Plant Biol. 10:34-40.

Wang, Xiaoyuan and Quinn, peter. J (2000).The location and function of vitamin E in membranes. Molecular Membrane Biology. 17:143- 156.

\section{How to cite this article:}

Bharati, S.P., Ravi Kant, Anil Kumar, V.K. Sharma and Choudhary, V.K. 2018. Study of Chemically Induced Variation in Agro-morphological Characters of Wheat Under Heat Stress. Int.J.Curr.Microbiol.App.Sci. 7(07): 1679-1691. doi: https://doi.org/10.20546/ijcmas.2018.707.198 